\title{
Experimental research on the penetration of tungsten-fiberl metallic-glass matrix composite material bullet into steel target
}

\author{
X.W. Chen ${ }^{\mathrm{a}}$ and G. Chen \\ Institute of Structural Mechanics, China Academy of Engineering Physics, Mianyang, Sichuan 621900, China
}

\begin{abstract}
In the present paper, the penetration experiments of tungsten-fiber/metallic-glass matrix composite material bullets into 45\# steel targets are conducted by employing H25 artillery. In which, an experimental technique of sub-caliber penetration is constructed. The quasi static and dynamic behaviours of tungsten-fiber/metallic-glass matrix composite material are also experimental investigated. The self-sharpening phenomenon of composite material is observed. Integrated with metallographic analysis, the failure modes of tungsten-fiber/metallic-glass matrix composite material are identified systemically and compared with the quasi-static and dynamic material tests. It includes four failure modes, i.e., shear fracture of tungsten fiber, brittle fracture of tungsten fiber and shear fracture of metallic glass matrix as well as melting of tungsten fiber and metallic glass matrix. Comparatively, three failure mechanisms of tungsten fiber in the bullet nose are also identified, i.e., shear fracture, splitting fracture and bending or/and buckling. Finally, the mechanism of self-sharpening behaviour of tungsten-fiber/metallicglass matrix composite material is discussed.
\end{abstract}

\section{Introduction}

Bulk metallic glass (BMG) is that, when the liquid alloy is cooled sharply to the glass transition temperature $T_{g}$, its microscopic structure freeze up to produce a noncrystalline alloy material. BMG has excellent mechanical, physical and chemist performances. One of its important features is, below the glass transition temperature $\left(T<T_{q}\right)$ or under condition of high stress/strain rate deformation, it is much easily to produce localized shear band. BMG has a high shear sensibility, and it may deduce a self-sharpening phenomenon at high speed impact with similar to indigenturanium. Therein BMG is possible to be employed in the new material of penetrator. That means, when the BMG penetrator impact the target plate, its nose will continually keep a sharp-pointed shape to improve the penetration capability. Comparatively, the nose of tungsten alloy penetrator may be blunted as mushroom shape during penetration.

However, the density of BMG is only $6 \mathrm{~g} / \mathrm{cm}^{3}$, which is quite smaller being as a penetrator material. Also, BMG almost has no macroscopic plasticity, and it restraints the application in penetration. Thus recently BMG composite material, e.g. tungsten-fiber/metallic-glass-matrix, has been developed rapidly. By introducing a second phase into $\mathrm{BMG}$, much more shear bands are induced to produce and slide. It guarantees the composite material still reserve BMG's performance, i.e., high strength, high hardness and shear self-sharpening, etc. Meanwhile, it reduces the macroscopic brittleness of material and remarkably increases the density.

Tungsten has high density, high strength and excellent ductility, and thus the tungsten-fiber/metallic-glass matrix composite material is employed to penetration. A large mount of research of tungsten-fiber/metallic-glass matrix composite material had been conducted recently, which covers material processing, experimental study and

a e-mail: chenxiaoweintu@yahoo.com theoretical analysis as well as numerical simulation. Conner et al [1] and Choi-Yim et al [2] conducted highspeed penetration tests to study the mechanical behaviour of tungsten-fiber/metallic-glass matrix material under high strain rate. Similar penetration tests were executed in NJUST [3,4]. Lei et al [5] also investigated the deformation and failure of tungsten-fiber/metallic-glass matrix composite material bullet in the conditions of normal/ oblique penetration or perforation.

In the present work, we conducted the penetration experiments of tungsten-fiber/metallic-glass matrix composite material bullets into $45 \#$ steel targets by employing H25 artillery. Self-sharpening phenomenon of bullet is observed by metallographic analysis and the material failure modes are identified systemically.

\section{The quasi-static and dynamic behaviours of tungsten-fiber/metallic-glass matrix composite material}

Instron1196 and SHPB are employed to conduct the experimental research on the quasi static and dynamic behaviours of tungsten-fiber/metallic-glass matrix composite material, respectively.

The loading rate of quasi-static material test is $0.2 \mathrm{~mm} / \mathrm{min}$ and its strain rate is $8.3 \times 10^{-4} \mathrm{~s}^{-1}$. Figure 1 (a) shows the stress-strain curves and the flow stress is about $2200 \mathrm{MPa}$. Some perfoliate cracks along the longitudinal direction are observed in the lateral side of the post-test specimens, as shown in figure 1(b). The impact velocity of SHPB tests vary between $11.7 \mathrm{~m} / \mathrm{s}$ and $16.7 \mathrm{~m} / \mathrm{s}$. Figure 2 shows the SHPB stress waves and dynamic stress-strain curve, respectively, at the loading rate of $12.3 \mathrm{~m} / \mathrm{s}$. Figure 3 shows the final deformation and fracture of specimens under different loading rates, and distinct cracking and destruction are observed.

In general, the dynamic behaviour of tungsten-fiber/ metallic-glass matrix composite material is depending on 


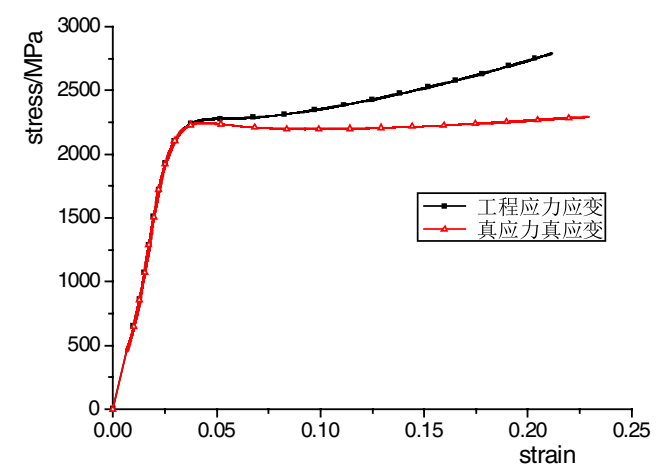

(a)

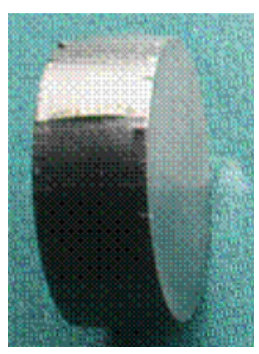

(b)

Fig. 1. Experimental results of quasi-static compression (a) stress-strain curve and (b) final deformation of specimen.

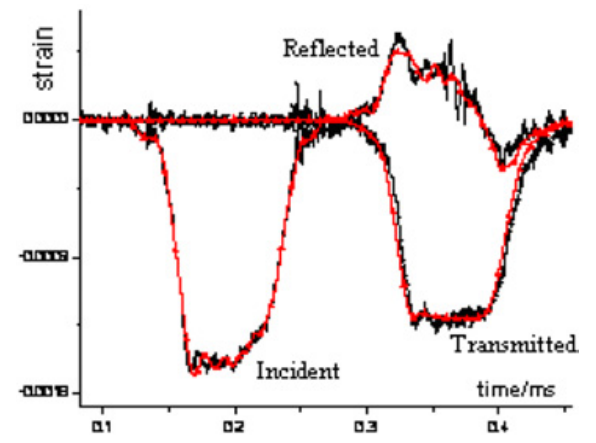

(a)

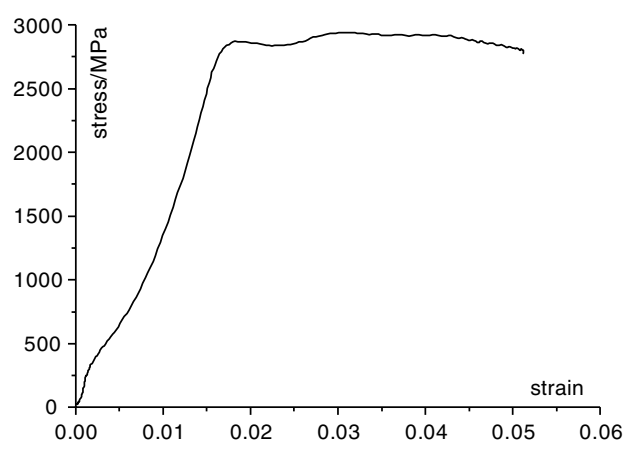

(b)

Fig. 2. Stress waves of SHPB and corresponding dynamic stressstrain curve (a) stress waves and (b) dynamic stress-strain curve.

the strain rate. Even at strain rate of $740 \mathrm{~s}^{-1}$, the dynamic flow stress is larger than $30 \%$ the quasi-static one. No hardening effect exists in the plastic flow stage under

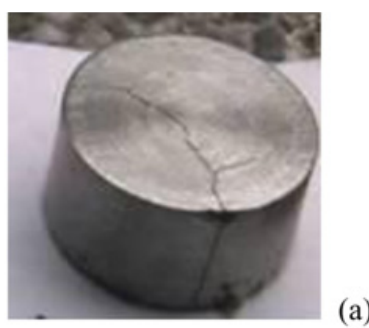

(a)

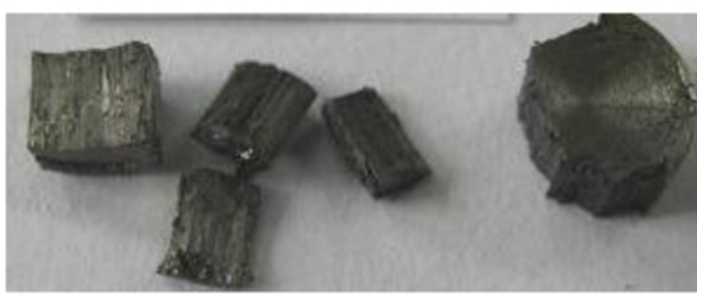

(b)

Fig. 3. Final deformation and fracture of specimens under different loading rates (a) $\mathrm{V}=12.3 \mathrm{~m} / \mathrm{s}$ and (b) $\mathrm{V}=16.7 \mathrm{~m} / \mathrm{s}$.

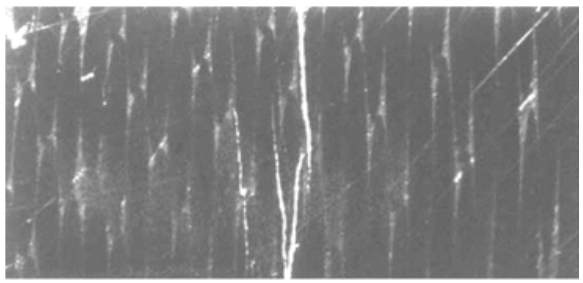

Fig. 4. Splitting and fracture of tungsten fibers in the specimen after SHPB experiment.

either quasi-static or dynamic loading. In macroscopic observation, the destruction pattern of SHPB specimen is likely as axial splitting. Comparatively, the microscopic failure includes the isolation of tungsten fiber and metallic glass matrix, splitting fracture of tungsten fiber, and so on.

\section{Penetration tests}

\subsection{Experimental installation and method}

Total 5 bullets of tungsten-fiber/metallic-glass matrix composite material are fired at impact velocities between $550 \mathrm{~m} / \mathrm{s}$ and $899 \mathrm{~m} / \mathrm{s}$. The bullet is cylindrical-like with a hemi-spherical nose, and its diameter and length are $7.5 \mathrm{~mm}$ and $50 \mathrm{~mm}$. respectively. The volume factor of tungsten fiber in the composite material is $80 \%$ and the material density is $17 \mathrm{~g} / \mathrm{cm}^{3}$.

Since the diameter of bullet is quite smaller than that of the H25 artillery, an experimental sub-caliber technique of APM2 bullet launched by the H25 artillery is employed in the present paper. A bakelite sabot is used to fit the larger diameter of artillery for firing a smaller bullet, which mass is much smaller than the bullet mass. In order to ensure a bullet impacts in good appearance, a bakelite rod is stick to the rear bottom of the bullet to move its centroid forward. The length of the bakelite rod is $15 \mathrm{~mm}$ and its mass is about $0.8 \mathrm{~g}$, while the bullet mass is $7.5 \mathrm{~g}$. Two impediment plates are mounted before the target plate to separate the sabot and stop the sabot fragments, respectively. 


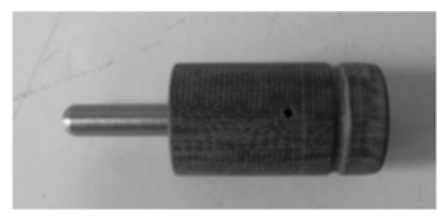

(a)

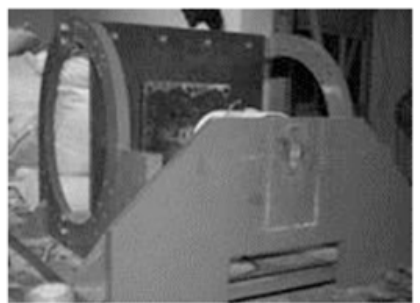

(b)

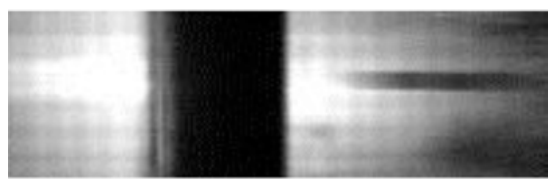

(c)

Fig. 5. Experimental technique of sub-caliber penetration (a) sabot and bullet, (b) target frame and (c) high-speed photograph.

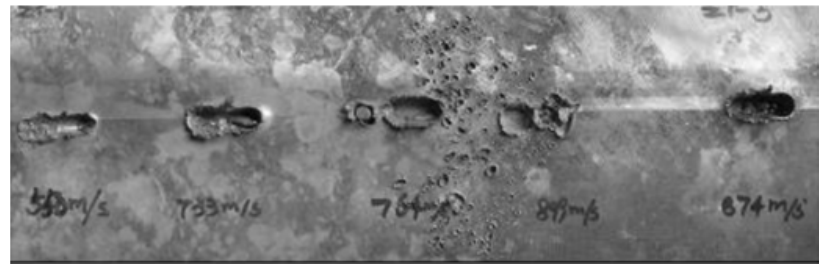

Fig. 6. 45\# steel target after penetration with different impact velocities.

The experimental installation is shown in figure 5(a)(b), and figure 5(c) shows the high-speed photograph of penetration. It is found that the bullet impacts $45 \#$ steel target in good appearance and no any yaw or deviation occurs. Meanwhile, the bullet completely separates from the bakelite sabot before it impacting the target.

\subsection{Macroscopic and microscopic observations of the experimental results}

The post-test target is shown in figure 6. In general, for most tests except No. 4 launch, the target surface around the crater is smooth, which indicate that the impediment plates are capable to stop the sabot fragments. However, at smaller impact velocities, e.g., $V=550 \mathrm{~m} / \mathrm{s}$ and $733 \mathrm{~m} / \mathrm{s}$, ricochet takes place and no effective penetration. Only under higher impact velocity, the bullets penetrate into the steel target effectively.

Figure 7 shows the macroscopically longitudinal section of $45 \#$ steel target after penetration in cases of $V=$ $874 \mathrm{~m} / \mathrm{s}$ and $V=764 \mathrm{~m} / \mathrm{s}$. The nose diameter of residual composite material bullet has no any obvious change. In particular, in case of $V=874 \mathrm{~m} / \mathrm{s}$, the bullet completely eroded, and it is unable to identify the original microscopic

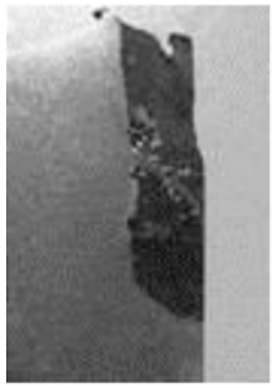

(a)

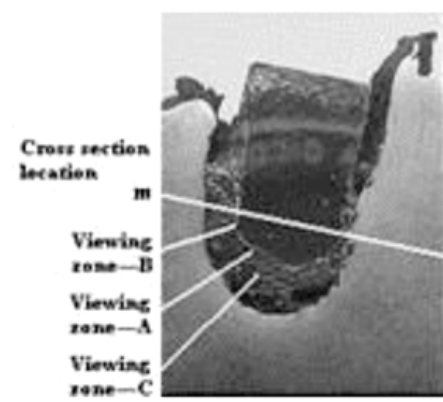

(b)
Fig. 7. Macroscopically longitudinal section of $45 \#$ steel target after penetration (a) $\mathrm{V}=874 \mathrm{~m} / \mathrm{s}$ and (b) $\mathrm{V}=764 \mathrm{~m} / \mathrm{s}$.

structure of tungsten fiber and metallic-glass matrix. There left a trace of reversal flowing of thawy bullet material along the ballistic path. In case of $V=764 \mathrm{~m} / \mathrm{s}$, the residual bullet includes two parts, i.e., eroding part and no deforming part. Also the trace of reversal flowing of thawy bullet material in the penetration hole is observed. The following analysis will mainly focus on the case of $V=764 \mathrm{~m} / \mathrm{s}$, to discuss the failure of bullet material under higher velocity impacting. The line $\mathrm{mn}$ in figure 7 (b) stands for the cross section location of the present analysis, and viewing zones $\mathrm{A}, \mathrm{B}$ and $\mathrm{C}$ are the corresponding location of analytical longitudinal section, respectively.

As shown in figure 7(b), after penetrating into \#45 steel plates, the nose shape of the tungsten-fiber/metallic-glass matrix composite material bullet deformed distinctly. The original hemi-spherical nose became as sharp nose due to the erosion, i.e., self-sharpening occurs. The diameter of ballistic hole in target always kept constant but the hole wall is quite rough and have the trace of highheat melting, friction and mass consumption. In the mean time, the self-sharpening of the bullet nose may cause the asymmetric resistant force acting on the bullet nose and therefore the ballistic locus of bullet deviated. The tungsten fibers, which located in the eroding part of bullet nose, shear fracture or/and bend along the direction with $60^{\circ}$ angle to the bullet axis. The tungsten fibers fall off from metallic-glass matrix after shear fracture. The shearing surfaces compose a sharp nose and it makes the bullet selfsharpening continually. By the way, the broken tungsten fibers stick on the lateral wall of ballistic hole of steel plate, and it seems as reversal flowing along the ballistic path.

Figure 8 are the metallographic graphs of transverse and longitudinal sections of initial composite material bullets, respectively. Obviously, in the transverse section, all the tungsten fibers arrange in order and proximately integrate with the metallic-glass matrix. The end section of tungsten-fiber is a standard circular shape. In the longitudinal section, the tungsten fiber direction agree with the bullet axis and the fibers look like slender ellipses, which since the longitudinal section crosses the bullet axis with a certain angle.

Figure 9 shows the metallographic graphs of left longitudinal section of bullet after penetration at velocity of $V=$ $764 \mathrm{~m} / \mathrm{s}$. The deformation and destruction of composite material bullet almost localizes in the margin of nose and its vicinity. In the zone of non-erosion and undeformation 


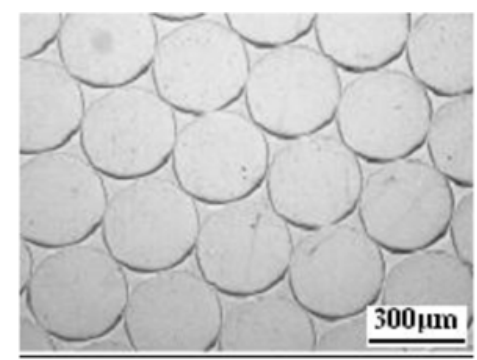

(a)

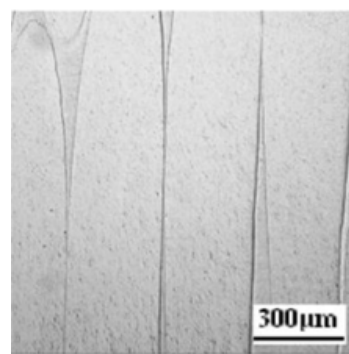

(b)

Fig. 8. Metallographic graphs of transverse and logitudinal sections of initial composite material penetrator (a) transverse section and (b) longitudinal section.

of bullet nose, tungsten fibers have no obvious plastic deformation, and its arranging direction is still parallel to the bullet axis. Meanwhile, metallic-glass matrix also has no any distinct damage. It indicates that the tungsten fiber and metallic-glass matrix in this zone are same as the initial material. Some melted metallic-glass matrix and tungsten fibers re-freeze and reserve in the ballistic hole of steel plate. The eroding material cumulates before the bullet nose and behaves as reversal flowing along the ballistic path. Melted tungsten fibers distribute without any order. It deduced that a large mount of heat come into being when the bullet penetrating into steel plate at higher velocity, which causes the metallic-glass matrix material soften and even molten. In the other hand, non-eroding bullet nose will reserve a clear outline contour with a certain acute angle, and all the tungsten fibers distribute in the same direction. The nose shape of the bullet displays a distinct characteristic of self-sharpen. It can be observed that splitting fracture exists in the part of non-eroding bullet material. Meanwhile, a remarkable disassociate gap occurs between the eroding part and non-eroding part.

\section{Metallurgical analyses of bullet nose}

We will conduct metallographic analyses on the transverse and longitudinal sections of bullet nose, to identify the different modes of self-sharpening shear failure of tungstenfiber/metallic-glass matrix composite material. All the selected zones and the corresponding illustrations are shown in figure 7(b).

\subsection{Analysis on the transverse sections of bullet nose}

With compared to figure 8(a), i.e., the metallographic graphs of the prototype bullet, at least 4 kinds of self-

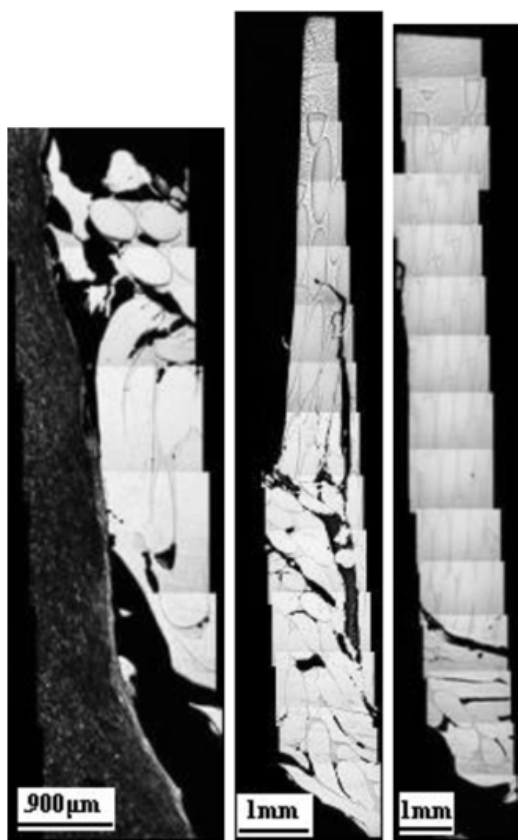

Fig. 9. Mettallographic graphs of left longitudinal section of bullet after penetration.
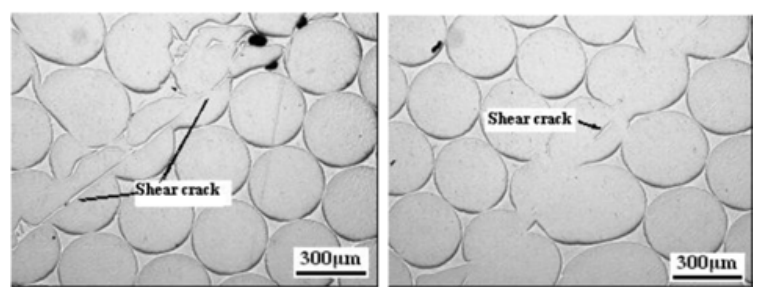

Fig. 10. Self-sharpening shear failure of bullet, Mode I: shear fracture of tungsten fibers.

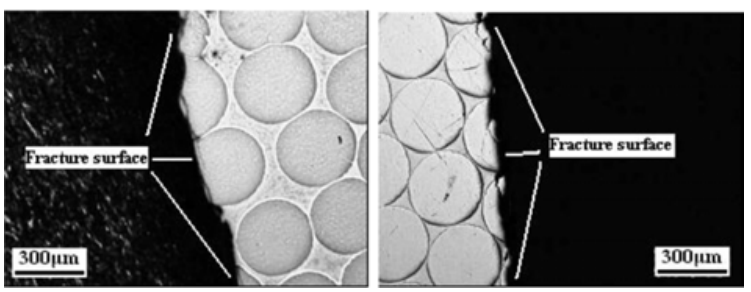

Fig. 11. Self-sharpening shear failure of bullet, Mode II: brittle fracture of tungsten fibers.

sharpening shear failure of tungsten-fiber/metallic-glass matrix composite material are identified as following:

Mode I: shear fracture of tungsten fibers. As shown in figure 10, the shear fracture will only arise in a row of tungsten fibers. Just in that narrow localization zone, the tungsten fibers melted and slipped to produce dislocation. Comparatively, the tungsten fibers around the shear band keep as intact as before. Therein the failure and destruction of material localize in a very narrow zone. It belongs to a typical adiabatic shear.

Mode II: brittle fracture of tungsten fibers. As shown in figure 11, it arise in the margin of bullet nose, accompanied with some fiber dreg. No any remarkable plastic deformation can be observed in the broken tungsten fiber. Similarly, 

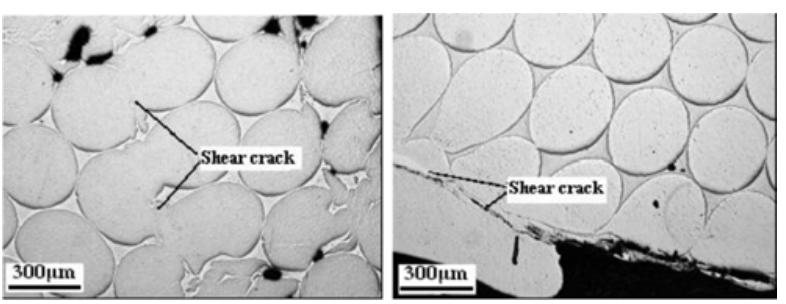

Fig. 12. Self-sharpening shear failure of bullet, Mode III: shear fracture of metallic glass matrix.

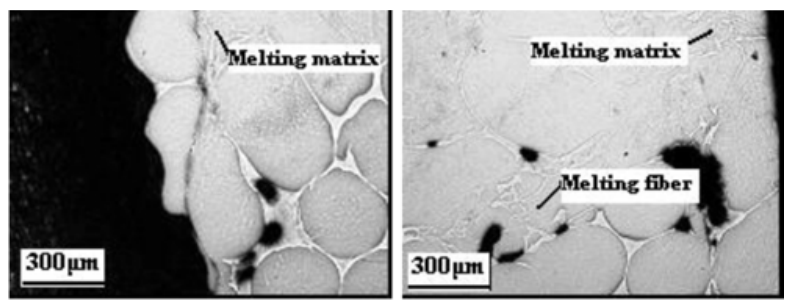

Fig. 13. Self-sharpening shear failure of bullet, Mode IV: melting of tungsten fibers and metallic glass matrix.

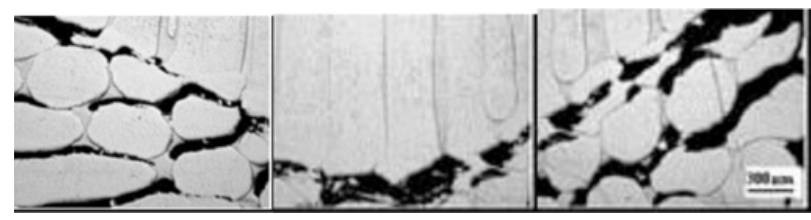

Fig. 14. Metallographic graph of the longitudinal section of penetrator nose after penetration.

the nearby tungsten fibers keep as intact as before. It is caused by scrape between bullet and steel target during high speed impact.

Mode III: shear of metallic glass matrix. As shown in figure 12, shear fracture occur in the metallic glass matrix, and cause the ambient tungsten fibers thermoplastic deform and flow along the shear direction. The metallic glass matrix detach from the tungsten fibers. All the deformation and failure are localized in a narrow zone.

Mode IV: melting of tungsten fibers and metallic glass matrix. As shown in figure 13, a large amount of heat arise in the high-speed penetration into the $45 \#$ steel plate of tungsten-fiber/metallic-glass matrix composite material bullets, which causes the metallic-glass matrix material and tungsten-fiber soften and even molten.

Most of the failure modes, except Mode II, are both related to the localized adiabatic heating. So far, we believe that higher temperature is the main reason to cause the failure of tungsten-fiber/metallic-glass matrix composite material at higher speed impact.

\subsection{Analysis on the longitudinal sections of bullet nose}

The self-sharpening phenomena may be observed clearly from the metallurgical graph shown in figure 14. Tungsten fiber breaks off and blunts at the nose tip of bullet, whilst it shears fracture in the lateral margin of nose and bends along the direction with $60^{\circ}$ angle to the bullet axis. The shearing surfaces compose a sharp nose and it makes the

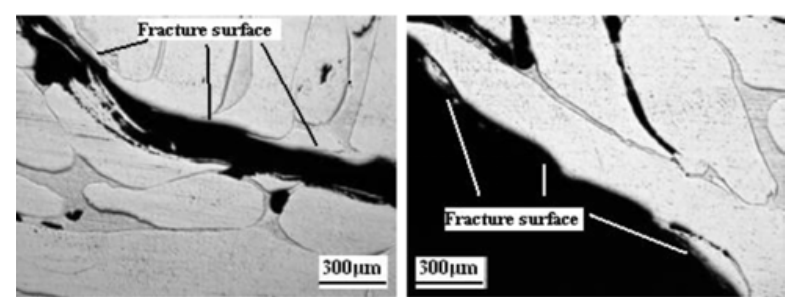

Fig. 15. Failure of tungsten fibers in the penetrator nose, mode I: shear fracture (Zone A).
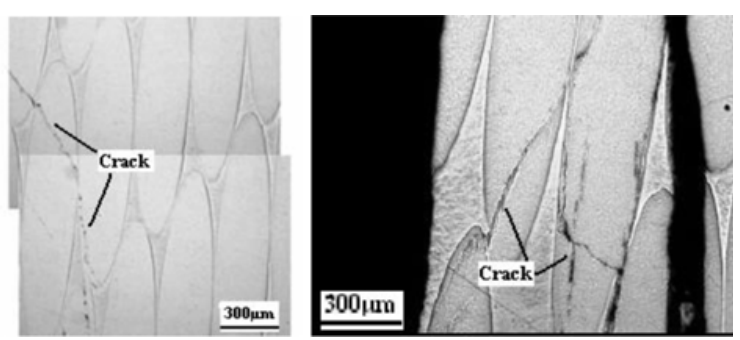

Fig. 16. Failure of tungsten fibers in the penetrator nose, mode 2 : splitting fracture (Zone B).

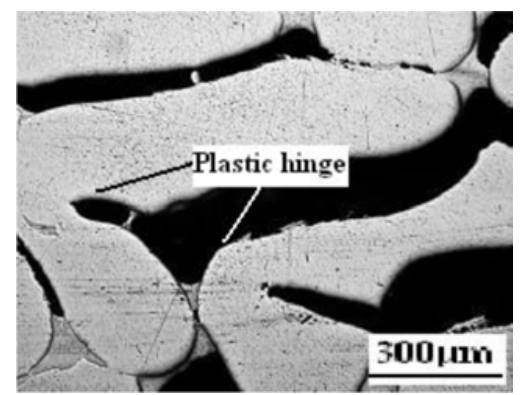

Fig. 17. Failure of tungsten fibers in the penetrator nose, mode 3 : bending and buckling (Zone $\mathrm{C}$ ).

bullet self-sharpening continually. In the non-eroding part of bullet nose, tungsten-fibers have no obvious plastic deformation, and its arranging direction is parallel to the bullet axis. Meanwhile, metallic-glass matrix also has no any remarkable damage. The tungsten-fiber and metallicglass matrix in this zone are consistent with the initial material. There is a remarkable disassociate gap between the eroding part and non-eroding part.

The eroding material cumulate up in front of the bullet nose and melted tungsten-fibers distribute without any order. Melted tungsten fiber display as different ellipse, and it indicate that fibre distribute not parallel to the bullet axis and arise transverse bending. Meanwhile, the metallic-glass matrix material had been soften and molten, and never adhesive to the tungsten-fiber. figure 14 also demonstrates that the broken fiber and matrix material divorce from the bullet nose continually due to the high speed impact and dynamic friction.

We will discuss the interaction of the mentioned failure modes. Figure 15-16 redisplay shear fracture and brittle splitting facture of tungsten fiber, respectively, while figure 17 shows the bending and buckling of tungsten fibers and it presents as a notable plastic hinge. In figure 15, shear fracture behaves as localization and it flows along 
the sharpening direction of bullet. Only a little tungstenfibers produce splitting fracture in figure 16 and the crack develop along the fiber distribution, which is similar to the cases of figure 1(b) and figure 3 observed in quasi-static and dynamic material test, respectively.

\section{Discussions}

As indicated in the above analysis, the destruction of the tungsten-fiber/metallic-glass matrix composite material bullets penetrating into $45 \#$ steel targets mainly includes localized shear and fracture, and localized adiabatic heating plays an important role on the material failure. Tungsten fibers not only enlarge the density of composite material projectile, but also increase the ductility and fracture strength of projectile material, and therein improve the penetration performance of bullet.

On the other hand, due to the limit of the maximum strain rate $\left(<10^{3} \mathrm{~s}^{-1}\right)$, only the isolation of tungsten fiber and metallic glass matrix, and the splitting fracture of tungsten fibers are observed in the SHPB tests. The experimental results are unable to coincide with the material failure modes under higher speed penetration. It is necessary to improve the SHPB technique to achieve the dynamic material test of high strength BMG and its composite under much higher strain rate.

Localized shear band is the most important characteristic of heterogeneous plastic deformation of BMG. The formation, evolution and interaction of the shear band dominate in the macroscopic mechanical behavior of BMG [6]. In particular, it is closely related to the self sharpening behavior of BMG. During the high-speed penetration, the plastic deformation of BMG and its composite may be localized intensively under higher temperature and high strain rate, and it is quite easy to transform as shear band. The formation of shear band may expand fast and induce material unstable fracture to execute self-sharpening.

In order to make use of self-sharpening effect of BMG and its composite, it is important to study the formation and evolution of the localized shear band. However, there are great arguments about the formation of localized shear band of bulk metallic glass. In general, viscosity decreases in some inhomogeneous location of BMG and further decreases the drag force of plastic deformation. This is the main reason to form the localized shear band. But, there are two entirely different presumptions about the viscosity reduction of $\mathrm{BMG}$, i.e., heat soften [7] and free-volume soften [8]. Recent research declares that the nucleation and evolution of shear band of BMG is a complex process of heat-force-free volume coupling [9]. Especially in the high speed penetration, the effect of localized adiabatic heating will be much more remarkable, and the interaction between free volume and heat will be further close together. Also, it is still unknown how to correlate between shear self-sharpen and shear band formation? Which factors affect the self-sharpening of
BMG and its composite? All these questions are worthy to be systemically studied.

\section{Conclusions}

The penetration experiments of tungsten-fiber/metallicglass matrix composite material bullets into $45 \#$ steel targets are conducted by employing $\mathrm{H} 25$ artillery and the self-sharpening phenomenon is observed. Integrated with metallographic analysis, the failure modes of composite material are identified and compared with the quasi-static and dynamic material test.

\section{Acknowledgements}

The project is supported by National Natural Science Foundation of China-NSAF (Grant No: 10976100). The tungsten-fiber/ metallic-glass matrix composite material is supplied by Dr. Fu HM, Institute of Metal Research, CAS.

\section{References}

1. Conner RD, Dandliker RB, Scruggs V, et al. Dynamic deformation behavior of tungsten-fiber /metallic-glass matrix composites. Int J Impact Engng, 2000, 24: 435-444.

2. Choi-Yim H, Conner RD, Szuecs F, et al. Quasistatic and dynamic deformation of tungsten reinforced Zr57Nb5A110Cu15.4Ni12.6 bulk metallic glass matrix. Scripta Materialia, 2001, 45: 1039-1045.

3. Rong G, Huang DW. Self-sharpening phenomena of tungsten fiber composite material penetrators during penetration. Explosion and Shock Waves, 2009, 29 (4): 351-355.

4. Rong G, Huang DW, Wang J. Research on penetration mechanism of tungsten fiber composite reinforced penetrators. Proceedings of 25th International Symposium on Ballistics, Beijing, China, 2010, pp: 1180-1186.

5. Lei B, Huang DW, Yang MC, et al. An experimental study on the self-sharpening behavior of amour piercer using tungsten fiber composite material. Transactions of Shenyang Ligong University, 2008, 27(1): 72-76.

6. Dai LH, Bai YL. Basic mechanical behaviors and mechanics of shear banding in BMGs. Int J Impact Engng, 2008, 34: 704-716.

7. Leamy H J, Wang T T, Chen H S. Plastic flow and fracture of metallic glass. Metallurgical Transactions, 1972, 3(3): 699-708.

8. Spaepen F. A microscopic mechanism for steady state inhomogeneous flow in metallic glasses. Acta Metallurgica, 1977, 25 (4): 407-415.

9. Jiang MQ, Dai LH. On the origin of shear banding instability in metallic glasses. J Mech Physics Solids, 2009; 57: 1267-1292. 